\title{
Desnutrición materna, bajo peso al nacer, pobreza y sociedad
}

\author{
Yessenia Eunice Recinos Méndez \\ Yessenia_recinos yahoo.es \\ Médico y Cirujana, Maestra en Ciencias en Educación y Ambientalización Curricular \\ Centro de Salud de Jalapa, Ministerio de Salud Pública y Asistencia Social de Guatemala
}

Fecha de recepción: 30 /11/2017 Fecha de aceptación: 05/02/2018

\begin{abstract}
Resumen
La salud integral de las embarazadas es un elemento importante para el desarrollo de un país. El estado nutricional de la gestante influye en el peso del recién nacido, lo que compromete la salud de los nuevos ciudadanos siendo determinantes críticos para la salud del individuo en su adultez. La malnutrición incrementa la morbi-mortalidad en las poblaciones, disminuye la capacidad productiva, la calidad de vida y aumenta los costos sociales, causando incremento de los cinturones de pobreza, lo que repercute en la población causando desigualdad y exclusión por falta de acceso a la salud, educación, empleo, saneamiento básico, perpetuando así el ciclo desnutrición y pobreza. Se realizó una búsqueda electrónica de la literatura sobre desnutrición materna, bajo peso al nacer y su relación a la pobreza y cómo influyen en el desarrollo de la sociedad haciéndose resumen y análisis de los artículos seleccionados. Se analizaron 38 publicaciones en las cuales se observó una relación estrecha entre la desnutrición materna, el bajo peso al nacer y pobreza. Conclusión: La desnutrición materna es un problema social ya que influye directamente en el desarrollo de los futuros ciudadanos; es importante incidir en ella positivamente para poder disminuir el riesgo de morbimortalidad perinatal e infantil que repercutirá negativamente en la salud de los futuros adultos e incrementa los costos sociales tanto individuales como colectivos.
\end{abstract}

\section{Palabras Clave}

Desnutrición materna, bajo peso al nacer, pobreza, desarrollo social

\begin{abstract}
The integral health of pregnant women is an important element for the development of a country, the nutritional status of the pregnant woman influences the weight of the newborn which compromises the health of the new citizens being critical determinants for the health of the individual in his adulthood. Malnutrition increases morbidity and mortality in populations, decreases productive capacity, quality of life and increases social costs, causing an increase in the poverty belts which affects the population causing inequality and exclusions due to lack of access to health, education, employment, basic sanitation, perpetuating the cycle of malnutrition and poverty. An electronic search of the literature on maternal malnutrition, low birth weight and its relation to poverty and how they influence the development of society was carried out by summarizing and analyzing the selected articles. We analyzed 38 publications in which a close relationship was observed between maternal malnutrition, low birth weight and poverty. Maternal malnutrition is a social problem since it directly influences the development of future citizens, it is important to influence it positively to reduce the risk of perinatal and infant morbidity and mortality that will negatively affect the health of future adults and increase social costs, both individual and collective.
\end{abstract}

\section{Keywords}

Maternal malnutrition, low birth weight, poverty, social development 


\section{Introducción}

La preocupación por mantener una vida saludable es tan antigua como la misma humanidad, el reconocer la interdependencia entre el desarrollo de la salud y el desarrollo social ha sido tan importante que desde la segunda mitad del siglo XX no hay discusión sobre la influencia de las relaciones sociales en la salud de la población (Berlinguer, 2007). El estado nutricional de todo individuo es de vital importancia para su desarrollo tanto individual como en la sociedad. Las alteraciones en el estado nutricional de la población es un tema de interés mundial cuando se sufre de los extremos de este, es decir obesidad y desnutrición.

La malnutrición incrementa la morbi-mortalidad en las poblaciones, disminuye la capacidad productiva, la calidad de vida y aumenta los costos sociales; siendo la desnutrición uno de los flagelos relevantes en el desarrollo de la humanidad es importante reconocer que es multifactorial, es decir que tiene componente genético, raza, etnia y social entre otros; causando incremento de los cinturones de pobreza, la población presenta desigualdad y exclusiones por falta de acceso a la salud, educación, empleo, saneamiento básico, perpetuando así la desnutrición en el mundo (Vallejo et al., 2016), lo que hace muy importante su estudio para la salud pública ya que tiene implicaciones trascendentales a nivel social y económico para los países.

Las gestantes no están ajenas a este problema ya que el estado nutricional alterado impacta negativamente los resultados maternos y perinatales, la salud materna y el desarrollo fetal son determinantes críticos para la salud del individuo en su adultez, por ejemplo hay estudios que evidencian la relación entre el bajo peso al nacer con enfermedades cardiovasculares posteriores (Velázquez et al., 2004), la desnutrición en embarazadas expone a un doble riesgo de bajo peso al nacer y este predispone de 2 a 10 veces más el riesgo de mortalidad neonatal, (Palmieri y Delgado, 2011), el bajo peso al nacer también es un predictor importante del desarrollo en el futuro del infante, se asocia directamente con la morbilidad y mortalidad infantil y se ha identificado que también es un factor de riesgo para la desnutrición proteico energética en niños menores de 1 año de edad (Velázquez et al., 1998).

Los recién nacidos con bajo peso al nacer tienen mayor riesgo de presentar problemas neuropsíquicos posteriores como: las malas adaptaciones al medio ambiente, alteraciones físicas y mentales que se pueden evidenciar en la edad escolar y pueden repercutir en la etapa adulta (Rodríguez et al., 2005). Si el estado nutricional de la madre es muy importante para los futuros ciudadanos de un país ¿Qué factores están influyendo para que este problema se esté presentando y cómo corregirlo?

\section{Metodología}

Se realizó una búsqueda electrónica de la literatura sobre desnutrición materna, bajo peso al nacer y su relación a la pobreza y cómo influyen en el desarrollo de la sociedad haciéndose resumen y análisis de los artículos seleccionados. Se analizaron varias publicaciones, en las cuales se observó una relación estrecha entre la desnutrición materna, el bajo peso al nacer y la pobreza.

\section{Referente teórico}

\subsection{Enfoque social}

El enfoque social está alcanzando cada vez más su verdadero espacio en el conocimiento y práctica social de la salud, lo cual ha logrado que surjan nuevas especialidades con el objetivo de que el aspecto social sea determinado en los procesos salud/enfermedad y cuyo desarrollo se vincula a procesos estructurales de la sociedad. A través de las ciencias sociales y sus instrumentos metodológicos se está logrando la comprensión del problema de salud de la población y se está avanzando en investigaciones en salud y la formación de recurso humano, es decir la medicina social radica en la incorporación de las ciencias sociales en el campo de la salud, operativizando los procesos de producción y reproducción social en las investigaciones, las políticas de educación en salud y los determinantes sociales en la salud (Palmieri y Delgado, 2011). 
El Programa de las Naciones Unidas para el Desarrollo (2015) define el desarrollo humano como: "la expresión de la libertad de las personas para vivir una vida prolongada, saludable y creativa; perseguir objetivos que ellas mismas consideren valorables; y participar activamente en el desarrollo sostenible y equitativo del planeta que comparten".

Con el paso de los años se ha hecho una lucha general para lograr avances científicos técnicos en materia de salud y cambios sociales (García y Suarez, 2007), teniéndose en los años 70 la declaración explícita de la OMS/OPS/UNICEF (1978) donde se indica "la necesidad de una estrategia integral que no solo preste servicios de la salud sino que también aborde las causas sociales, económicas y políticas subyacentes en la mala salud".

Pero las metas trazadas no han sido logradas, el siglo XXI se inició sin ver cumplida la meta de la Conferencia de Alma-Ata: Salud para todos en el 2000. Por su parte, en la Declaración de la Cumbre del Milenio, se encuentran objetivos, metas e indicadores relacionados con salud (Torres y Mujica, 2004), aunque se lograron algunos avances importantes en los objetivos de desarrollo del milenio (SEGEPLAN, 2010), las metas no fueron alcanzadas como se esperaba, los avances en la tecnología y la práctica están condicionados por cada contexto social en su momento histórico, siendo estas limitadas a las formas específicas de conocimiento de cada época y al desarrollo social (García, 2011), lo cual da desigualdad entre los avances en diferentes países y abre más la brecha existente entre ellos.

Según Hertzman et al. (2001), hay determinantes sociales que influyen en el desarrollo, definiendo tres niveles, siendo estas: el macro ambiente socioeconómico nacional, donde el determinante salud es el ingreso per cápita y su distribución equitativa, el meso ambiente: el nivel de la sociedad civil o de ambiente donde se dan factores como redes, normas y realidades sociales que hacen posible la cohesión y las capacidades de las instituciones para responder a las necesidades humanas y el micro ambiente o determinantes sociales de la salud asociados con las personas.
América Latina enfrenta grandes retos de cara a los desafíos globales, entre ellos los planteados en la Agenda 2030 para el Desarrollo Sostenible (Girón, 2016a) ya que se espera tener una transformación en la forma de ver y abordar el desarrollo, la cual llegue a atender las necesidades de las personas, con esperanzas de lograr avanzar y superar dichos objetivos; la región latinoamericana cuenta con grandes recursos como la agricultura, la minería, el petróleo, cuencas hidrográficas, etc., las cuales pueden potencializar su productividad de manera racional y mejorar las estrategias políticas económicas para disminuir la brecha que existe entre los países desarrollados por el bien de la población (Álvarez, 2016), pero se deberá tener cuidado de no comprometer las generaciones posteriores dándoles un mal uso a dichos recursos.

Se espera que al disminuir las distancias existentes en las desigualdades entre los países, al interior de los mismos, entre mujeres y hombres, así como el cuidado del medio ambiente, se logre un cambio en la forma en el qué y cómo se produce el mejoramiento y la salvaguarda del bien público, así como elevar los ingresos a partir del empleo digno (Girón, 2016b) y que esto repercuta positivamente en el estado de salud de la población.

La falta de empleo, el empleo en condiciones precarias, el estancamiento económico, la volatilidad financiera y monetaria, la pobreza y la inequidad que van contra los trabajadores, son problemas que influyen en el desarrollo de las sociedades, las políticas públicas que imperan para superar la crisis están agravando las situaciones de los espacios laborales, (Girón, 2016 b)

Es importante tomar en cuenta que el manejo macroeconómico nacional e internacional muchas veces confunde los objetivos con las herramientas, algunos economistas tienen como metas aumentar el crecimiento económico, disminuir la inflación, aumentar el empleo y la productividad, etc., sin tomar en cuenta que el objetivo fundamental de todo sistema económico es que todas las personas vivan con condiciones dignas, que les permitan mejorar cada vez más su calidad de vida (Durán, 2007) y disminuir la vulnerabilidad social existen- 
tes, la cual depende de factores relacionados con el nivel de salud de las personas, la edad y las condiciones higiénicas, ambientales y la ubicación territorial donde residen (ENSMI, 2015) poniendo a algunos grupos de la población en mayor riesgo que otros y que inciden directamente en la salud dada su incapacidad de resistencia cuando se presentan eventos negativos.

Según la economía clásica y neoclásica el mejorar las condiciones de vida depende de las característica macroeconómicas pero muchas de las evidencias empíricas muestran que los países pueden tener crecimiento durante largos periodos de tiempo sin disminuir la pobreza estructural de la población, es decir existe un punto en el que el aumento del crecimiento no logra disminuir las condiciones de pobreza de la población (Duran, 2007).

\subsection{Pobreza}

El fenómeno de la pobreza es muy complejo y multidimensional, siendo esta producto de la imposibilidad de tener acceso o carencia de los recursos para satisfacer tanto psíquica como físicamente las necesidades básicas humanas que inciden en el nivel y calidad de vida de las personas, tales como: la alimentación, la vivienda, la educación, la asistencia en salud y el acceso al agua potable, asociado a ello se agregan las relaciones con la cultura, las capacidades y la dinámica de las fuerzas sociales y la falta de oportunidades, lo cual hace mucho más complejo el concepto de pobreza (CIEN, 2010).

Según López (2007), existe la pobreza estructural explicándola a través de las llamadas trampas de la pobreza, en las cuales lamentablemente tras generaciones las familias no logran salir de los círculos viciosos de pobreza con retroalimentación de condiciones que empeoran su situación. Aunque estas trampas son apenas una consecuencia en que se derivan las causas reales de la pobreza estructural, dichas trampas pueden afectar a nivel individual como: el trabajo infantil, el analfabetismo, la desnutrición, la criminalidad, entre otros, o pueden afectar a nivel regional como: físicas y geográficas, ambientales, desplazamiento forzado, etc.
Para la UNICEF (2015), la reducción de la pobreza y la pobreza extrema ha sido muy limitada en los últimos 25 años y en Guatemala no se lograron alcanzar los objetivos del milenio, lamentablemente la pobreza con frecuencia se define por el ingreso monetario como la medida unidireccional, pero este no debe ser el único indicador que la defina ya que existen múltiples aspectos que la constituyen, siendo estos factores, entre otros: mala salud, falta o poca educación, insuficiente nivel de vida, falta de ingresos, falta de poder tomar decisiones, malas condiciones de trabajo, la violencia, desnutrición, etc., por lo cual, no sólo los recursos materiales sino también los emocionales y espirituales son necesarios para vivir, desarrollarse y prosperar (UNICEF, 2005).

Según el Banco Mundial (2009), entre 2000 y 2006 Guatemala pudo reducir la pobreza en 5 puntos, de 56 a $51 \%$, lo cual es un logro positivo, sin embargo si se ve más allá la realidad es que la pobreza moderada disminuyó pero la extrema aumentó debido a que el alza de los alimentos ha sido mayor que el incremento general de los precios, llevando al costo de la canasta básica a un aumento tal que hace que los niveles de consumo de los extremadamente pobres no se logren realizar, lo cual hace que este grupo de población esté más expuesto a desnutrición junto a otras enfermedades.

Para el 2006 UNICEF (2015), indicó que la proporción de los niños y niñas de Guatemala se encontraban en pobreza multidimensional en un $79.9 \%$ siendo esta la segunda más alta en América Latina y el Caribe, si los niños y niñas se encuentran en este nivel de pobreza, esto indica que las familias en general se encuentran en este estado tan lamentable, incluyendo a las madres.

Se ha visto que la alta tasa de natalidad es más común en las personas con bajo nivel económico, los hogares más grandes tienden a ser más pobres, ya que a medida que aumenta el número de integrantes en el hogar el consumo per cápita disminuye por cada hijo a un 19\% (CIEN, 2010). La fecundidad es una variable demográfica importante para evaluar la tendencia del crecimiento poblacional, el aumento de esta tiene repercusiones negativas 
en la disminución de la mortalidad materna infantil y el volumen total de la población (ENSMI, 2015).

\subsection{Embarazo y bajo peso}

A diferencia de otros países en los cuales la edad fértil es considerada de 14 a 44 años, en Guatemala se establece de los 10 a los 54, ya que las mujeres inician la edad reproductiva tempranamente y la finalizan tardíamente asociado a la no aceptación de métodos de planificación familiar, esto hace que las familias sean más numerosas y con menores recursos, el embarazo es fisiológico pero expone a la mujer, al feto y al recién nacido a la probabilidad de enfermar o morir, siendo esta probabilidad mayor si se presenta desnutrición en la madre o en el recién nacido, (Donoso et al., 2014). Los embarazos antes de los 20 años y después de los 35 traen como consecuencia una mayor probabilidad de muerte materna y perinatal y la muerte materna afecta a la comunidad completa, ya que en general es la madre la encargada del cuidado de los hijos mientras los padres trabajan y esto repercute en el desarrolla de la familia, ya que al momento de faltar la madre generalmente son las hijas mayores las que tienen que tomar a su cargo el hogar abandonando el estudio y tomando responsabilidades que no van de acuerdo a su madurez psíquica y fisiológica.

Los estados de malnutrición afectan a casi el 50\% de mujeres en edad fértil y preescolar, los que han sido resultado de modelos de desarrollo imperantes en los cuales no se incluye a grupos importantes de la población siendo la malnutrición expresión de la inseguridad alimentaria y problema para el crecimiento y desarrollo nacional (Palmieri y Delgado, 2011).

La salud integral de las embarazadas es un elemento crucial para el desarrollo de un país. El embarazo presenta características únicas desde el punto de vista alimentario y nutricional. El estado nutricional de la mujer embarazada tanto antes y durante el embarazo es definitivamente un factor fundamental para la salud de ella y su hijo (Mendoza y Sánchez, 2010). Asociado también el embarazo puede desencadenar otras enfermedades como las relacionadas a hipertensión arterial, la diabetes gestacional, entre otras, que pueden predisponer a que se presenten partos prematuros que complican a la madre y al bebé (Ministerio de Salud de la Nación, 2012).

El embarazo constituye la etapa de mayor vulnerabilidad nutricional en la vida de la mujer por el aumento de la actividad anabólica que hace que las necesidades nutricionales sean mayores (Díaz et al., 2013). La relación entre la masa corporal de la madre y el desarrollo del producto es directa, las mujeres que inician el embarazo con índice de masa corporal menor de $19.8 \mathrm{Kg} / \mathrm{m}$ (bajo peso) presentan más peligros para sus productos (Rodrieguez et al., 2011).

Según Grados et al., (2003), el estado nutricional pregestacional de la madre y la ganancia de peso durante la gestación son los dos factores relacionados con el peso al nacer y este con la morbimortalidad perinatal, el crecimiento antropométrico y el desarrollo mental del recién nacido.

Si existe un estado nutricional alterado en la madre se tendrá el riesgo de presentar un intercambio inadecuado materno-fetal, es decir un metabolismo anormal de proteínas, lípidos, carbohidratos y minerales en la madre propiciaran un desarrollo inadecuado en los fetos por falta de los nutrientes (Prendes et al., 2001), por lo cual es necesario que toda mujer embarazada tenga un control prenatal oportuno y preciso estableciendo un diagnóstico nutricional veraz para implementar medidas adecuadas y así efectuar un cambio positivo en ellas (Mendoza et al., 2010), aunque el periodo del embarazo no es un momento óptimo para corregir la desnutrición (Cedillo et al., 2006) se ha demostrado que las intervenciones nutricionales destinadas a promover un normal crecimiento intrauterino reduce el riesgo de bajo peso al nacer y la incidencia de parto prematuro que es otro factor de riesgo muy importante (Arcos et al., 1995). Es indispensable identificar el estado nutricional materno pregestacional y durante la misma con el fin de reconocer las pacientes que tienen mayor probabilidad de complicarse (Romano et al., 2008). 
La edad de la madre también es otro factor importante con el peso al nacer (Chaviano y López, 2000), las madres adolescentes tienen la mayor incidencia de recién nacidos de bajo peso en relación a otros grupos etarios, por lo que el embarazo en adolescentes constituye otro problema social, económico y de salud pública (Cedillo et al., 2006). La población mundial de adolescentes ha aumentado, siendo esta población de más de mil millones de habitantes, una de cada cuatro personas en los países en vías de desarrollo está entre 10 y 19 años de edad, a diferencia de una de cada siete personas en los países desarrollados (Uzcátegui, 2002).

El embarazo en adolescentes es un problema relevante en la salud reproductiva en el mundo, cada año aproximadamente 15 millones de adolescentes dan a luz, lo cual corresponde a una quinta parte de todos los nacimientos (Romano et al., 2008) siendo la población de adolescentes tan alta, la salud integral del adolescente debe de ser un elemento crucial para el desarrollo de un país.

La población de menores de 19 años en Guatemala para el 2015 se estimaba en 56\%, la tasa global de fecundidad disminuyó de un 5.7 en el quinquenio del 1985-1990 a un 3.7 en 2010-2015 (UNICEF, 2015).

De acuerdo con el informe elaborado por la Defensoría de la Mujer, de la Procuraduría de los Derechos Humanos informa que en el 2010 se reportaron 45 mil 48 partos en niñas, adolescentes y mujeres jóvenes y que 1 de cada 5 niños y niñas nacen de una madre adolescente entre 15 y 19 años de edad. (PDH, 2013)

Según Cedillo et al., (2006), las madres adolescentes presentan con mayor frecuencia problemas psicosociales y familiares que muchas veces están asociados a otras situaciones, tales como familias disfuncionales, bajo rendimiento escolar, conductas depresivas, abuso de sustancias, maltratos, y embarazos ocultos, lo cual no permite identificar tempranamente los factores de riesgo asociados a la edad para el bajo peso al nacer de sus hijos, muchas adolescentes pueden sufrir rechazo familiar y social, Ilevándola a conflictos psicológicos, crean- do sensaciones de insatisfacción, que repercuten en no asumir el rol de la maternidad afectando directamente el desarrollo de sus hijos y vulnerando su estado de salud.

Otro factor importante que influye en el bajo peso al nacer es el nivel de educación de las mujeres, se estima que la educación y la salud están íntimamente unidas, y que las niñas con mayor nivel educativo tienen menos hijos y los tienen más tardíamente, lo que las hace mejores madres ya que tienen un nivel de madurez mayor (Chan, 2010).

El ciclo de la pobreza, la enfermedad y la persistencia intergeneracional del bajo nivel económico de la mujer se puede romper a través de darles educación, brindándoles oportunidades igual que a los hombres, las mujeres alfabetizadas tienen mayor conocimiento sobre salud, regularmente contribuyen a los ingresos familiares, lo que las hace invertir en el bienestar de su familia. El papel que las mujeres han desempeñado en los últimos diez años al integrarse en el mercado laboral es crucial ya que la disminución de la pobreza y la desigualdad en los países de América Latina y el Caribe han sido notables debido a esta participación( Azevedo et al., 2012).

Mirta Roses (2003), directora de la Organización Panamericana de la Salud dijo: "La integración entre salud y educación es uno de los motores fundamentales del desarrollo de la década", además aseguró que cuanto menor sea el nivel educativo son menos las oportunidades que se tendrán para poder ingresar en el mercado laboral, los ingresos a los hogares son menores, lo que hace que se tengan carencias en relación a vivienda, bienes y servicios. Además el nivel educativo de la madre tiene influencia en la desnutrición crónica de los niños y los niveles de mortalidad infantil.

Según el informe de la Procuraduría de los Derechos Humanos en Guatemala en el 2013 las perspectivas de pobreza eran desalentadoras, puesto que la juventud tenía poco acceso a la educación. Para ese año el $49.46 \%$ de los jóvenes estaban en pobreza y el $14 \%$ en pobreza extrema, factor que influye a que los adolescentes abandonen los estudios para tener que entrar en el mercado laboral 
tempranamente y las adolescentes optan por tener uniones tempranas, lo que las hace entrar en el círculo de madres adolescentes, poca educación, bajo peso al nacer y desnutrición infantil.

\section{Conclusiones}

Por lo anterior se puede concluir que son múltiples los factores que influyen en el desarrollo social y económico de los seres humanos.

La importancia que han tomado las ciencias sociales en la salud ha ayudado a darle un enfoque más amplio a las enfermedades no solo investigándolo desde sus causas científicas sino también en el entorno sociocultural.

Es importante actuar sobre determinantes sociales de la salud con criterios de integración, equidad, solidaridad y reducción de las brechas de inequidad a través de definir y promover políticas intersectoriales.

Le educación y la salud van de la mano contribuyendo a ofrecerle a las personas la oportunidad de desarrollar plenamente su potencial y lograr salir de la pobreza, siendo fundamental para lograr la reducción de dichas brechas e inequidades sociales existentes.

La malnutrición de la población no es solo debido a la pobreza, esta va de la mano con los niveles de educación tan limitados y a la herencia generacional de los diferentes patrones económicos que se han vivido, donde los modelos del liberalismo, neoliberalismo, entre otros, no han alcanzado los beneficios para todos sino solo para unos pocos.

Los factores que interactúan para determinar el desarrollo y resultado final del embarazo son múltiples, pero la desnutrición materna y su nivel de estudio influye directamente en el bienestar del recién nacido.

La desnutrición de las mujeres embarazadas no solo las afecta a ellas y a los recién nacidos, sino a la población en general, ya que esto predispone a que en el futuro las poblaciones sean más vulnerables teniendo mayor mortalidad infantil e individuos con mayores problemas de salud, lo que repercute en mayor gasto económico y más compromiso social.

Las embarazadas adolescentes están en mayor riesgo de sufrir desnutrición y tener hijos con bajo peso al nacer, si no tienen un nivel mínimo de educación también las hace más vulnerables ya que desconocerán cómo deben criar a sus hijos lo que aumentará el riesgo de morbilidad y mortalidad neonatal e infantil.

\section{Referencias bibliográficas}

Álvarez, A. (2016) Retos de América Latina: Agenda para el Desarrollo Sostenible y Negociaciones del siglo XXI Problemas del Desarrollo. Revista Latinoamericana de Economía, vol. 47, núm. 186, julio-septiembre, pp. 9-30

Arcos, E. et al., (1995) Relación entre el estado nutricional de madres adolescentes y el desarroIlo neonatal. Bol Oficina Sanit Panam vol.118, no. 6, pp. $488-498$

Azevedo, J. et al., (2012) Embarazo adolescente y oportunidades en América Latina y el Caribe, sobre la maternidad temprana, pobreza y logros económicos.

Banco Mundial, (2009) Guatemala, Evaluación de la pobreza, buen desempeño a bajo nivel. Informe No. 43920-GT 18 de marzo, Departamento América Central, Unidad de Reducción de Pobreza y Gestión Económica, Región de América Latina y el Caribe.

Berlinguer, G. (2007) . "Determinantes sociales de la enfermedad". Rev Cubana Salud. Vol. 33, no. 1.

Cedillo, N. et al. (2006), Estado nutricional de las adolescentes embarazadas: relación con el crecimiento fetal. Rev Obstet Ginecol Venez vol.66 no. 4. 
Chan, M. (2010) La educación y la salud están intimamente unidas. Cumbre sobre los Objetivos de Desarrollo del Milenio. Intervención en la segunda mesa redonda, sobre la consecución de los objetivos relacionados con la salud y la educación, Nueva York (Estados Unidos de América) 20 de septiembre.

Chaviano, J. y D. López. (2000) Edad materna, riesgo nutricional preconcepcional y peso al nacer. Rev Cubana Alimen Nutr. vol.14, no. 2):94-99.

CIEN (Centro de Investigaciones Económicas Nacionales) (2010) Diagnóstico de la pobreza en Guatemala, lineamientos de política económica, social y de seguridad 2012-2020, Guatemala, noviembre.

Díaz, M. et al., (2013) Consejos útiles sobre la alimentación y nutrición de la embarazada. Manual para los profesionales de la Salud. Editorial Lazo Adentro, 2013.

Donoso, E. et al., (2014) La edad de la mujer como factor de riesgo de mortalidad materna, fetal, neonatal e infantil. Rev. Med. Chile; vol. 142, pp. $168-174$

Durán, J. (2007). La pobreza y su relación con el desarrollo social, el Crecimiento. Nuevas Hipótesis Teóricas de Implicaciones Prácticas. Conferencias Banco Central de Colombia, Julio.

ENSMI. (2015) VI Encuesta Nacional de Salud Materno Infantil ENSMI 2014-2015 Informe de Indicadores Básico, Guatemala, noviembre 2015.

García, R. y R. Suárez, (2007) La educación terapéutica: Propuesta de un modelo teórico desde la experiencia del Programa Cubano de Educación en Diabetes. Washington: OPS.

García, R. (2011) El enfoque social como elemento esencial del que hacer por la salud de la población. Revista Cubana de Salud Pública. vol. 37 pp. 675-685.
Girón, A. (2016a) Objetivos del desarrollo sostenible y la agenda 2030: Frente a las políticas y los cambios de gobierno de América Latina. Problemas del Desarrollo. Revista Latinoamericana de Economía, vol. 47, núm. 186, julio-septiembre, pp. 3-8.

Girón, A. (2016b) Sembrando ideas. Aportaciones a una teoría del desarrollo desde el Sur Problemas del Desarrollo. Revista Latinoamericana de Economía, vol. 47, núm. 184, enero-marzo, pp. 3-6.

Grados, F. et al., (2003) Estado nutricional pregestacional y ganancia de peso materno durante la gestación y su relación con el peso del recién nacido. Rev Med Hered. vol. 14, no. 3, pp.128-132.

Hertzman C, et al., (2001) Using an interactive framework of society and life course to explain self-rated health in early adulthood. Soc. Sci Med.; vol, 53, pp. 1575-1585.

López, H. y J. Nuñez, (2007). La pobreza y la desigualdad en Colombia. Departamento Nacional de Planeación, Bogotá D.C, Colombia.

Mendoza, L. et al., (2010) Estado nutricional de embarazadas en el último mes de gestación y su asociación con las medidas antropométricas de sus recién nacidos Pediatr. (Asunción), Vol. 37; no 2. Pp.91-96.

Ministerio de Salud de la Nación. (2012) Nutrición y Embarazo. Recomendaciones en Nutrición para los equipos de salud-Dirección Nacional de Maternidad e Infancia. Buenos Aires.

OMS/OPS/UNICEF. (1978) Conferencia Internacional sobre Atención Primaria de Salud de Alma-Atá, URSS, 6-12 de septiembre.

Palmieri, M. y H. Delgado (2011). Análisis situacional de la malnutrición en Guatemala sus causas y abordaje. Programa de las Naciones Unidas para el Desarrollo,(PNUD) p 42. 
PDH (Procuraduría de los Derechos Humanos) (2013) Análisis de la Situación de embarazadas en niñas y adolescentes en Guatemala 2011-2013 Informe Temático, Guatemala, octubre.

PNUD (2015) Programa de las Naciones Unidas para el Desarrollo y sus Informes de Desarrollo Humano en Chile 2015.

Prendes, M. et al., (2001). Estado nutricional materno y peso al nacer. Rev. Cubana Med Gen Integr . vol. 17 no. 1, pp. 35-42

Rodríguez, D. et al., (2005). Bajo peso al nacer: Algunos factores asociados a la madre. Revista Cubana de Obstetricia y Ginecología, vol. 31 no. 1.

Rodríguez, D. et al., (2011). Implicaciones obstétricas de la desnutrición materna. Revista Médica vol. 33 no. 4 , pp. 448-455..

Romano, M. et al., (2008) Desnutrición en gestantes adolescentes y su relación con bajo peso. Revista de Posgrado de la Vla Cátedra de Medicina. N ${ }^{\circ} 187$ - Noviembre pp. 1-5.

Roses, M. (2003) Discurso de toma de posesión directora OPS . Rev Panam Salud Publica Vol 13, pp. 2-3.

SEGEPLAN (2010) Tercer informe de avances en el cumplimiento de los Objetivos de desarrollo del milenio. Objetivo 5: Mejorar la salud materna Guatemala. Serviprensa, Guatemala, noviembre.
Torres, C. y Mujica OJ. (2004) Salud, equidad y los objetivos de desarrollo del milenio. Rev. Panam Salud Pública. Vol. 15, no. 6, pp. 430-39.

UNICEF (2015) Convención derechos de la niñez, Principales avances y desafíos del Estado y el pueblo de Guatemala en el cumplimiento de los derechos de la infancia con motivo de los 25 años de la Convención sobre los Derechos del Niño. Primera edición.

Uzcátegui O. (2001) Embarazo en la adolescente y en la añosa Caracas. Editorial Ateproca; pp. 211-216.

Vallejo, S. et al., (2016) "Estado nutricional y determinantes sociales en niños entre 0 y 5 años de la comunidad de Yunguillo y de Red Unidos, Mocoa, Colombia." Rev Uni. Salud. Vol.18, no.1, pp. 113-125.

Velázquez, A. et al., (1998). Factores de riesgo de desnutrición proteico-energética en niños menos de 1 año de edad. Rev. Cubana Aliment Nutr vol. 2 no. 2 pp. 82-5.

Velázquez, Q. et al., (2004). Recién nacidos con bajo peso; causas, problemas y perspectivas a futuro. Boletín médico del Hospital Infantil de México, vol. 61, no.1, pp 73-86. 
